\title{
Back-to-Back Isolated Photon-Quarkonium Production at the LHC and the Transverse-Momentum-Dependent Distributions of the Gluons in the Proton
}

\author{
J. P. Lansberg \\ IPNO, Université Paris-Sud, CNRS/IN2P3, F-91406, Orsay, France \\ Jean-Philippe.Lansberg@in2p3.fr
}

Published 29 February 2016

\begin{abstract}
The study of isolated heavy quarkonia, such as $J / \psi$ and $\Upsilon$, produced in association with a photon in proton-proton collisions at the LHC, is probably the optimal way to get right away a first experimental determination of two gluon transverse-momentumdependent distribution (TMDs) in an unpolarized proton, $f_{1}^{g}$ and $h_{1}^{\perp g}$, the latter giving the distribution of linearly polarized gluons. To substantiante this, we calculate the transverse-momentum-dependent effects that arise in the process under study and discuss the feasibility of their measurements.
\end{abstract}

Keywords: TMDs, quarkonium, LHC.

\section{Introduction}

It is nowadays well recognized that an important class of observables at particle colliders are transverse ${ }^{\mathrm{a}}$-momentum distributions of final state events. While a transverse-momentum $\left(q_{T}\right)$ distribution can be described within the standard collinear factorization approach for large $q_{T}$, it is convenient and sometimes necessary to resort to an alternative factorization approach for small $q_{T}$, typically $q_{T} \sim M_{p} \ll Q, M_{p}$ being the proton mass and $Q$ the hard scale of the process under investigation. This alternative approach, called Transverse-Momentum-Dependent (TMD) factorization (see e.g. Ref. 1), takes into account the transverse motion of partons w.r.t. the direction of the parent proton. In this picture the small finalstate transverse momentum $q_{T}$ is a consequence of the parton transverse momenta. Hence, the non-perturbative distribution functions entering a TMD factorization

This is an Open Access article published by World Scientific Publishing Company. It is distributed under the terms of the Creative Commons Attribution 3.0 (CC-BY) License. Further distribution of this work is permitted, provided the original work is properly cited.

${ }^{\mathrm{a}}$ with respect to the beam axis 
formula not only depend on the collinear momentum fractions $x$, but also on the transverse momentum $p_{T}$.

The information on the transverse-momentum dependence of unpolarized and linearly polarized gluons in an unpolarized proton is encapsulated in the following TMD correlator $\Phi_{g}$, which describes the transition from a proton to a gluon, such that

$$
\begin{array}{r}
\Phi_{g}^{\mu \nu}\left(x, \boldsymbol{p}_{T}, \zeta, \mu\right) \equiv 2 \int \frac{\mathrm{d}(\xi \cdot P) \mathrm{d}^{2} \xi_{T}}{(x P \cdot n)^{2}(2 \pi)^{3}} e^{i\left(x P+p_{T}\right) \cdot \xi} \\
\operatorname{Tr}_{c}\left[\left\langle P\left|F^{n \nu}(0) \mathcal{U}_{[0, \xi]}^{n[-]} F^{n \mu}(\xi) \mathcal{U}_{[\xi, 0]}^{n[-]}\right| P\right\rangle\right]_{\xi \cdot P^{\prime}=0} \\
=-\frac{1}{2 x}\left\{g_{T}^{\mu \nu} f_{1}^{g}-\left(\frac{p_{T}^{\mu} p_{T}^{\nu}}{M_{p}^{2}}+g_{T}^{\mu \nu} \frac{\boldsymbol{p}_{T}^{2}}{2 M_{p}^{2}}\right) h_{1}^{\perp g}\right\}
\end{array}
$$

The gluon four-momentum $p$ is decomposed as $p=x P+p_{T}+p^{-} n$, where $n$ is a lightlike dimensionless vector, conjugated to the momentum $P$ of the parent proton, with no transverse components and satisfying the relation $\zeta^{2}=(2 n \cdot P)^{2} / n^{2}$ (one assumes an analogous decomposition for $k$ and one takes $\left.p^{-}=k^{+}=0\right), p_{T}^{2}=-\boldsymbol{p}_{T}^{2}$ and $g_{T}^{\mu \nu}=g^{\mu \nu}-P^{\mu} n^{\nu} / P \cdot n-n^{\mu} P^{\nu} / P \cdot n$. In addition, $F^{\mu \nu}(x)$ is the gluon field strength. At leading twist, $\Phi_{g}$ is parametrized ${ }^{7}$ in terms of the two gluon TMD distributions discussed above, $f_{1}^{g}$ and $h_{1}^{\perp g}$. In Eq. (1), the gauge link $\mathcal{U}_{[0, \xi]}^{n[-]}$ is needed to render the matrix element gauge invariant. It runs from 0 to $\xi$ via minus infinity along the $n$ direction.

A model-independent positivity bound for $h_{1}^{\perp g}$ was derived in Ref. 7 and reads

$$
\frac{\boldsymbol{p}_{T}^{2}}{2 M_{p}^{2}}\left|h_{1}^{\perp g}\left(x, \boldsymbol{p}_{T}^{2}\right)\right| \leq f_{1}^{g}\left(x, \boldsymbol{p}_{T}^{2}\right) .
$$

Since it is $T$-even, $h_{1}^{\perp g}$ does not necessarily vanish in absence of initial and final state interactions. This does not prevent it to be nonuniversal if it receives contributions from such interactions.

A number of suggestions to measure these unknown TMDs, $f_{1}^{g}$ and $h_{1}^{\perp g}$, have been discussed in the literature. Whereas $h_{1}^{\perp g}$ can, in principle, be extracted from the azimuthal dependence of the dijet production in $p p$ collisions, ${ }^{4}$ TMDfactorization breaking effects may be significant in this case due to the existence of both initial and final state interactions. ${ }^{5}$ To avoid these, one may prefer to rely on heavy-quark or dijet electro-production ${ }^{2,3}$ which would however only be studied at a future EIC facility. Diphoton production ${ }^{6}$ should also not be sensitive to factorization-breaking effects. It however suffers from a huge $\pi^{0}$ background and from contaminations from quark-induced channels at RHIC energies which may preclude a clean gluon TMD extraction. It has recently been proposed ${ }^{10}$ to look at $C=+1$ quarkonium production in the region where their transverse momentum is smaller than their mass. A one-loop analysis has shown that ${ }^{1} S_{0}$ production may be 
safer ${ }^{13}$ than that of ${ }^{3} P_{J}$ where color-octet contributions might spoil factorization. ${ }^{13}$ Low- $q_{T} C=+1$ quarkonium production may however be very challenging at the LHC; a first study of $\eta_{c}$ production has just been carried out by the LHCb but for $q_{T}>6 \mathrm{GeV}$. Prospects are certainly more promising with the proposed LHC fixed-target experiment AFTER@LHC(see e.g. Ref. 14, 15, 16, 17).

We claim here that polarized and unpolarized gluon TMDs can be accessed right now at the LHC through the reaction ${ }^{18}$

$$
p\left(P_{A}\right)+p\left(P_{B}\right) \rightarrow \mathcal{Q}\left(P_{\mathcal{Q}}\right)+\gamma\left(P_{\gamma}\right)+X
$$

where now $\mathcal{Q}$ is one of the $C=-1$ charmonium or bottomonium (e.g. $J / \psi$ or $\Upsilon$ ) produced almost back-to-back with a photon. In this case, the momentum imbalance of the pair in the final state, $\boldsymbol{q}_{T}=\boldsymbol{P}_{\mathcal{Q}_{T}}+\boldsymbol{P}_{\gamma T}$, is small, but not the individual transverse momenta of the two particles. These should be well detectable by the ATLAS or CMS detectors for instance. As we discussed in Ref. 18, both these final states are always produced by gluon fusion (this is in fact also true at lower energies ${ }^{16}$ ), as illustrated by the thin and thicker curves on Fig. 1. In this particular configuration, the process $\Upsilon+\gamma$ is expected to be dominated by the color-singlet contributions $^{19}$ (see the solid blue and dashed orange curves on Fig. 1), hence TMD factorization should be applicable since the final-state interactions are expected to be suppressed. In the case of $J / \psi+\gamma$, a precise assessement of a possibe colorsinglet dominance depends a more precise knowledge of color octet NRQCD matrix elements, which is still lacking. ${ }^{20-22}$ To be on the safe side and to single out the color-singlet contribution, it could be useful to isolate the $J / \psi$. It may also be useful to measure $J / \psi+\gamma$ production (see Ref. 23 and references therein) in general in order to check the possible contribution from Double-Parton Scatterings (DPS).

It has however recently been emphasised in an analysis of Higgs plus jet production at the $\mathrm{LHC}^{24}$ that it may not be necessary to restrict the analyses to final states made only of colorless particles for the TMD factorisation to be applicable, provided that one of the particles used to measure the momentum imbalance is colorless. If this is the case, color-octet contributions to quarkonium + photon would not break the TMD factorisation. By extension, the study of the momentum imbalance in $J / \psi$ plus jet could also be used to extract gluon TMDs. At this point, let us mention that back-to-back $J / \psi+Z$, which has been studied by ATLAS, ${ }^{25}$ could also be used to extract gluon TMDs. The arguments in favor of this observable are that it would probe them at higher scales $\left(Q \simeq m_{Z}\right)$ and provide us with information on their evolution (see Ref. 29), that in disfavor is the very small expected yield. ${ }^{30}$ On the contrary, $J / \psi+W$ is likely contaminated by quark-induced contributions. ${ }^{31}$ In addition, both $J / \psi+Z$ and $J / \psi+W$ are likely be contaminated by significant DPS contributions ${ }^{25,32}$; a careful study of their suppression by the smallmomentum-imbalance requirement would therefore be needed. The same proviso holds for quarkonium-pair production. ${ }^{26-28}$ 


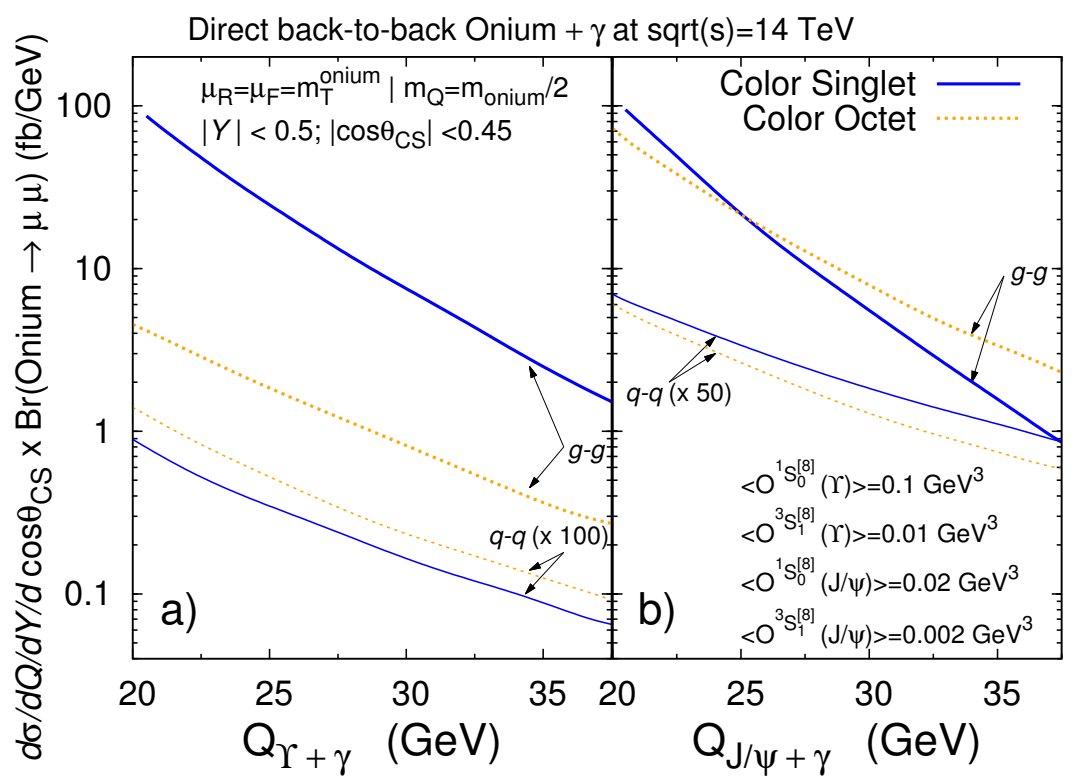

Fig. 1. Color-octet and color-singlet contributions from $g-g$ fusion and $q-\bar{q}$ annihilation channels to the production of a photon with a) an $\Upsilon$ and b) a $J / \psi$ as a function of $Q$, the invariant mass of the pair. The curves for the $q-\bar{q}$ annihilation are respectively rescaled by a factor 100 (50) in a) (b)).

\section{TMD Formalism for Photon-Quarkonium Production}

In the TMD-factorization approach, the cross section for near back-to-back $\mathcal{Q}+\gamma$ production is given by

$$
\begin{aligned}
\mathrm{d} \sigma=\frac{1}{2 s} \int & \mathrm{d} x_{a} \mathrm{~d} x_{b} \mathrm{~d}^{2} \boldsymbol{p}_{T, a} \mathrm{~d}^{2} \boldsymbol{p}_{T, b} \frac{\mathrm{d}^{3} \boldsymbol{P}_{\mathcal{Q}} \mathrm{d}^{3} \boldsymbol{P}_{\gamma}}{16 \pi^{2} E_{\mathcal{Q}} E_{\gamma}} \delta^{4}\left(p_{a}+p_{b}-P_{\mathcal{Q}}-P_{\gamma}\right) \\
& \times \operatorname{Tr}\left\{\Phi_{g}\left(x_{a}, \boldsymbol{p}_{T, a}, \zeta, \mu\right) \Phi_{g}\left(x_{b}, \boldsymbol{p}_{T, b}, \zeta, \mu\right)|\mathcal{A}(g g \rightarrow \mathcal{Q} \gamma)|^{2}\right\}
\end{aligned}
$$

where $s=\left(P_{A}+P_{B}\right)^{2}$ is the total energy squared in the hadronic centre-of-mass frame. We stress that the $q_{T}$ dependence of the cross section is completely factored out from the hard-scattering amplitude squared $|\mathcal{A}(g g \rightarrow \mathcal{Q} \gamma)|^{2}$.

One then finds that

$$
\begin{aligned}
& \frac{\mathrm{d} \sigma}{\mathrm{d} Q \mathrm{~d} Y \mathrm{~d}^{2} \boldsymbol{q}_{T} \mathrm{~d} \Omega}=\frac{4 \alpha_{s}^{2} \alpha^{2} e_{Q}^{2}\left|R_{0}(0)\right|^{2}}{3 M_{\mathcal{Q}}^{2}} \frac{Q^{2}-M_{\mathcal{Q}}^{2}}{s Q^{3}\left(\left(\gamma^{2}+1\right)^{2}-\left(\gamma^{2}-1\right)^{2} \cos ^{2} \theta\right)} \times \\
& \times\left\{F_{1} \mathcal{C}\left[f_{1}^{g} f_{1}^{g}\right]+F_{3} \mathcal{C}\left[w_{3} f_{1}^{g} h_{1}^{\perp g}+\left(x_{a} \leftrightarrow x_{b}\right)\right] \cos 2 \phi+F_{4} \mathcal{C}\left[w_{4} h_{1}^{\perp g} h_{1}^{\perp g}\right] \cos 4 \phi\right\}
\end{aligned}
$$

where $Q$ and $Y$ are, respectively, the invariant mass and the rapidity of the pair, $x_{a, b}=\exp [ \pm Y] Q / \sqrt{s}$ and the solid angle $\Omega=(\theta, \phi)$ is measured 
in the Collins-Soper frame $\mathrm{b}^{\mathrm{b}}$ and $\mathcal{C}[w f g] \equiv \int \mathrm{d}^{2} p_{T, a} \int \mathrm{d}^{2} p_{T, b} \delta^{2}\left(p_{T, a}+p_{T, b}-\right.$ $\left.\boldsymbol{q}_{T}\right) w\left(p_{T, a}, p_{T, b}\right) f\left(x_{1}, p_{T, a}^{2}\right) g\left(x_{2}, p_{T, a}^{2}\right)$ In Eq. (5), $R_{0}(0)$ is the quarkonium radial wave function at the origin in the position space and $e_{Q}$ is the heavy-quark charge in units of the proton charge. The factors $F_{i}$ are given by $F_{1}=1+2 \gamma^{2}+9 \gamma^{4}+$ $\left(6 \gamma^{4}-2\right) \cos ^{2} \theta+\left(\gamma^{2}-1\right)^{2} \cos ^{4} \theta, F_{3}=4 \gamma^{2} \sin ^{2} \theta$ and $F_{4}=\left(\gamma^{2}-1\right)^{2} \sin ^{4} \theta$ with $\gamma \equiv Q / M_{\mathcal{Q}}$. The explicit expressions for the transverse weights are

$$
w_{3}=\frac{\boldsymbol{q}_{T}^{2} \boldsymbol{p}_{b T}^{2}-2\left(\boldsymbol{q}_{T} \cdot \boldsymbol{p}_{b T}\right)^{2}}{2 M_{p}^{2} \boldsymbol{q}_{T}^{2}}, w_{4}=2\left[\frac{\boldsymbol{p}_{a T} \cdot \boldsymbol{p}_{b T}}{2 M_{p}^{2}}-\frac{\left(\boldsymbol{p}_{a T} \cdot \boldsymbol{q}_{T}\right)\left(\boldsymbol{p}_{b T} \cdot \boldsymbol{q}_{T}\right)}{M_{p}^{2} \boldsymbol{q}_{T}^{2}}\right]^{2}-\frac{\boldsymbol{p}_{a T}^{2} \boldsymbol{p}_{b T}^{2}}{4 M_{p}^{4}}
$$

\section{Extraction of $f_{1}^{g}$ and $h_{1}^{\perp g}$}

By measuring the following $q_{T}$-dependent observables,

$$
\mathcal{S}_{q_{T}}^{(n)} \equiv \frac{\int \mathrm{d} \phi \cos (n \phi) \frac{\mathrm{d} \sigma}{\mathrm{d} Q \mathrm{~d} Y \mathrm{~d}^{2} \boldsymbol{q}_{T} \mathrm{~d} \Omega}}{\int_{0}^{Q^{2} / 4} \mathrm{~d} \boldsymbol{q}_{T}^{2} \int \mathrm{d} \phi \frac{\mathrm{d} \sigma}{\mathrm{d} Q \mathrm{~d} Y \mathrm{~d}^{2} \boldsymbol{q}_{T} \mathrm{~d} \Omega}},
$$

with $n=0,2,4$, one can single out the three terms in Eq. (5). We obtain

$\mathcal{S}_{q_{T}}^{(0)}=\frac{\mathcal{C}\left[f_{1}^{g} f_{1}^{g}\right]}{\int \mathrm{d} \boldsymbol{q}_{T}^{2} \mathcal{C}\left[f_{1}^{g} f_{1}^{g}\right]}, \mathcal{S}_{q_{T}}^{(2)}=\frac{F_{3} \mathcal{C}\left[w_{3} f_{1}^{g} h_{1}^{\perp g}+\left(x_{a} \leftrightarrow x_{b}\right)\right]}{2 F_{1} \int \mathrm{d} \boldsymbol{q}_{T}^{2} \mathcal{C}\left[f_{1}^{g} f_{1}^{g}\right]}, \mathcal{S}_{q_{T}}^{(4)}=\frac{F_{4} \mathcal{C}\left[w_{4} h_{1}^{\perp g} h_{1}^{\perp g}\right]}{2 F_{1} \int \mathrm{d} \boldsymbol{q}_{T}^{2} \mathcal{C}\left[f_{1}^{g} f_{1}^{g}\right]}$

Fig. 2 shows predictions for $\mathcal{S}_{q_{T}}^{(0,2,4)}$ for the process $\Upsilon+\gamma$ with different Ansätze for the TMD distributions. We find that the size of $\mathcal{S}_{q_{T}}^{(0)}$ should be sufficient to allow

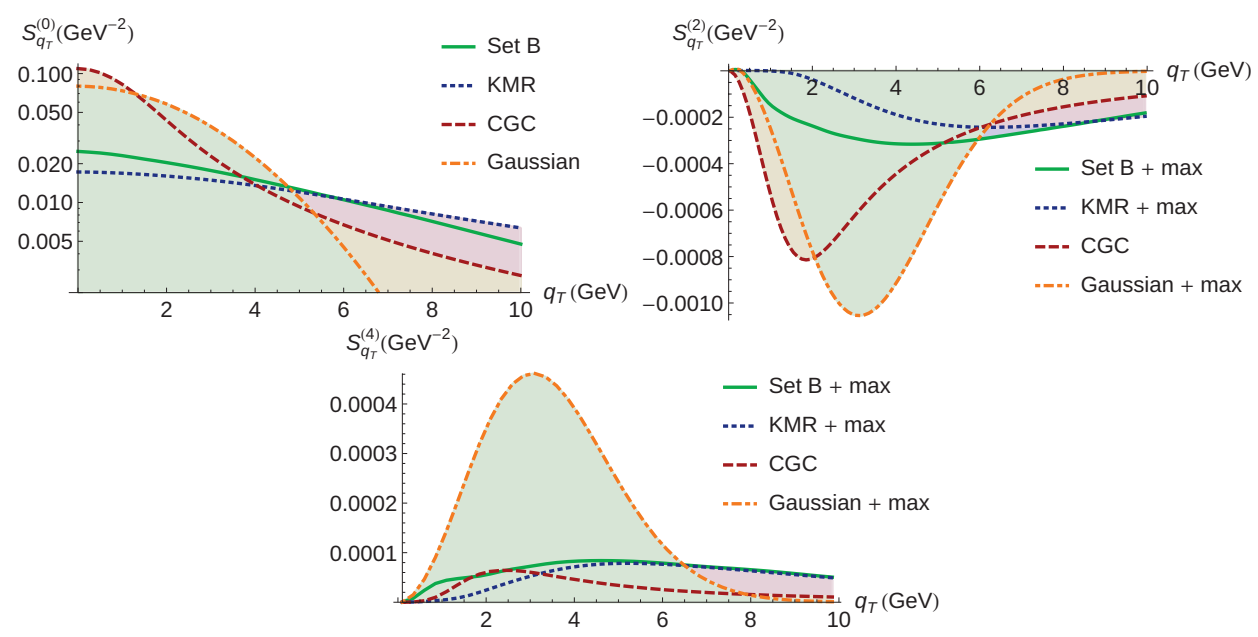

Fig. 2. Evaluation of the azimutally-modulated $q_{T}$ spectra $\mathcal{S}_{q_{T}}^{(0)}, \mathcal{S}_{q_{T}}^{(2)}$ and $\mathcal{S}_{q_{T}}^{(4)}$ given in Eq. (8) for the process $p p \rightarrow \mathcal{Q} \gamma X$, for $\sqrt{s}=14 \mathrm{TeV}, Q=20 \mathrm{GeV}, Y=0, \theta=\pi / 2$.

${ }^{\mathrm{b}}$ The Collins-Soper frame is such that the pair is at rest and the $\hat{x} \hat{z}$-plane spanned by $\left(\boldsymbol{P}_{A}, \boldsymbol{P}_{B}\right)$ and the $\hat{x}$-axis set by their bisector. ${ }^{33}$ 
for an extraction of $f_{1}^{g}$ as a function of $q_{T} . \mathcal{S}_{q_{T}}^{(2)}$ and $\mathcal{S}_{q_{T}}^{(4)}$ are small and one would need to integrate them over $\boldsymbol{q}_{T}^{2}$, (up to $Q^{2} / 4$ ), to look for an experimental evidence of a nonzero $h_{1}^{\perp g}$.

\section{Conclusion}

We claim that a first experimental determination of the polarized and unpolarized gluon TMD distributions for $x$ on the order of $10^{-3}$ can be obtained from the analyses of azimuthal asymmetries and transverse-momentum spectra in $p p \rightarrow J / \psi(\Upsilon) \gamma X$ at the LHC. The yields are large enough to perform these analyses using already existing data at the center-of-mass energies $\sqrt{s}=7$ and $8 \mathrm{TeV}$.

\section{Acknowledgments}

I would like to thank Wilco den Dunnen, Cristian Pisano and Marc Schlegel with whom I have collaborated on the topic presented here. This work was supported in part by the French CNRS, Grants No. PICS-06149 Torino-IPNO and No. PEPS4AFTER2.

\section{References}

1. J.C. Collins, Foundations of Perturbative QCD, (Cambridge University Press, Cambridge, 2011); S.M. Aybat, T.C. Rogers, Phys. Rev. D83, 114042 (2011); J.C. Collins, T.C. Rogers, Phys. Rev. D87, 034018 (2013); M.G. Echevarria, A. Idilbi, I. Scimemi, JHEP 1207, 002 (2012).

2. D. Boer, S.J. Brodsky, P.J. Mulders and C. Pisano, Phys. Rev. Lett. 106, 132001 (2011).

3. C. Pisano, et al., JHEP 1310, 024 (2013).

4. D. Boer, P.J. Mulders and C. Pisano, Phys. Rev. D 80, 094017 (2009).

5. T.C. Rogers and P.J. Mulders, Phys. Rev. D 81, 094006 (2010).

6. J.W. Qiu, M. Schlegel and W. Vogelsang, Phys. Rev. Lett. 107, 062001 (2011).

7. P.J. Mulders and J. Rodrigues, Phys. Rev. D 63, 094021 (2001).

8. D. Boer, et al., Phys. Rev. Lett. 108, 032002 (2012).

9. D. Boer, W.J. den Dunnen, C. Pisano, M. Schlegel, Phys. Rev. Lett. 111, 032002 (2013).

10. D. Boer and C. Pisano, Phys. Rev. D 86 (2012) 094007

11. J. P. Ma, J. X. Wang and S. Zhao, Phys. Rev. D 88 (2013) 014027

12. R. Aaij et al. [LHCb Collaboration], arXiv:1409.3612 [hep-ex].

13. J.P. Ma, J.X. Wang and S. Zhao, Phys. Lett. B 737 (2014) 103

14. S.J. Brodsky, F. Fleuret, C. Hadjidakis, J.P. Lansberg, Phys. Rept. 522 (2013) 239

15. J.P. Lansberg, S.J. Brodsky, F. Fleuret, C. Hadjidakis, Few Body Syst. 53 (2012) 11

16. J. P. Lansberg, et al. EPJ Web of Conf. 85, 02038 (2015)

17. L. Massacarier et al., these proceedings.

18. W. J. den Dunnen, J. P. Lansberg, C. Pisano, M. Schlegel, Phys. Rev. Lett. 112 (2014) 212001

19. P. Mathews, K. Sridhar and R. Basu, Phys. Rev. D 60, 014009 (1999).

20. N. Brambilla, et al. Eur. Phys. J. C 71 (2011) 1534

21. J. P. Lansberg, Eur. Phys. J. C 61 (2009) 693 
22. J. P. Lansberg, Int. J. Mod. Phys. A 21 (2006) 3857

23. J. P. Lansberg, Phys. Lett. B 679 (2009) 340

24. D. Boer and C. Pisano, arXiv:1412.5556 [hep-ph].

25. G. Aad et al. [ATLAS Collaboration], arXiv:1412.6428 [hep-ex].

26. J. P. Lansberg and H. S. Shao, Phys. Rev. Lett. 111 (2013) 122001

27. G. P. Zhang, Phys. Rev. D 90 (2014) 9, 094011

28. J. P. Lansberg and H. S. Shao, arXiv:1410.8822 [hep-ph].

29. D. Boer and W. J. den Dunnen, Nucl. Phys. B 886 (2014) 421

30. B. Gong, J. P. Lansberg, C. Lorce and J. Wang, JHEP 1303 (2013) 115

31. J. P. Lansberg, C. Lorce, Phys. Lett. B 726 (2013) 218; erratum Ibid. 738 (2014) 529

32. G. Aad et al. [ATLAS Collaboration], JHEP 1404 (2014) 172

33. J.C. Collins and D.E. Soper, Phys. Rev. D 16, 2219 (1977). 